Archive for

Organic Chemistry

Arkivoc 2018, part v, 0-0

\title{
The study of regioselectivity of ferrocenylalkylation of N,S- heterocycles in aqueous-organic media
}

Elena Yu. Rogatkina (Osipova) ${ }^{a}$, Anna S. Ivanovab, Alexey N. Rodionova, Alexander S. Peregudova, Alexander A. Korlyukov ${ }^{a}$, Alexander D. Volodin ${ }^{a}$, Yury A. Belousov, ${ }^{a}$ and Alexander A. Simenel*a,c

${ }^{a}$ A.N. Nesmeyanov Institute of OrganoElement Compounds, Russian Academy of Sciences, 28 Vavilov St., 119991 Moscow, Russian Federation

${ }^{b}$ Federal State Budget Educational Institution of Higher Education «Moscow Technological University», 78 Vernadsky prospect, 119454 Moscow, Russian Federation

"National University of Science and Technology "MISIS", Chemistry Department, 4 Leninskiy prospekt, 119049 Moscow, Russian Federation

Email: alexsim@ineos.ac.ru

Received 11-27-2017

Accepted 05-06-2018

Published on line 07-03-2018

\section{Abstract}

Regioselectivity of ferrocenylalkylation reaction of mercaptoheterocycles with ferrocenylcarbinoles in biphasic aqueous-organic media was studied. The structures of compounds were assigned on the basis of NMR spectra and ${ }^{1} \mathrm{H} /{ }^{13} \mathrm{C}$ heteronuclear correlations. X-ray determination of molecular structures of 1(ferrocenyl(phenyl)methyl)pyrrolidine-2-thione 5f, 4,5-dihydro-1-(1-ferrocenylmethyl)-1H-imidazole-2-thiol 7a and 4,5dihydro-1-(1-ferrocenyl-2methylpropyl)-1H-imidazole-2-thiol 7e were carried out.

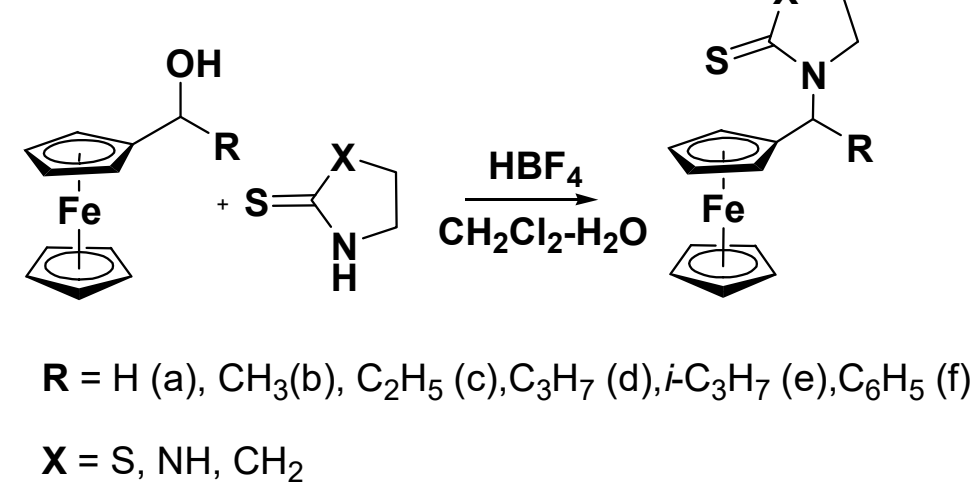

Keywords: Ferrocene, ferrocenylalkylation, mercaptoheterocycles, regioselectivity, X-Ray analysis 


\section{Introduction}

Ferrocene-containing heterocyclic compounds with great success have proved themselves as potential medicines for the treatment of diseases of various origin. ${ }^{1-3}$ The presence of an antitumor effect in heterocyclic ferrocene derivatives is most relevant, because an effective and low-toxic anti-cancer drug has not yet been invented. Numerous in vivo and in vitro tests carried out by our group together with biologists have revealed a pronounced antitumor cytostatic effect of heterocyclic ferrocene derivatives. ${ }^{4-8}$ Moreover together with biologists from Lomonosov Moscow State University (Russian Federation) identified a sonodynamic effect (activity under the influence of ultrasound) of ferrocenylheterocyclic porphyrins was revealed in relation to the cells of $S$. aureus (Staphylococcus aureus). ${ }^{9}$

A convenient synthetic method for the production of heterocyclic ferrocene derivatives is the alkylation of azoles by hydroxy(alkyl)ferrocenes ( $\mathrm{FcCH}(\mathrm{R}) \mathrm{OH})(\mathbf{1}$ a-e) in a two-phase aqueous-organic system (watermethylene chloride) during the catalysis with a strong mineral acid with a non-oxidizing agent $\left(\mathrm{HBF}_{4}\right)$. Previously the alkylation of unsymmetrically substituted heterocycles with ferrocenylcarbinols was carried out. Our team reported recently that the reaction proceeded regioselectively if there was a significant difference in the electronic effects of the substituents on a heterocycle. ${ }^{10}$ However, the regioselectivity of the alkylation of ambident nucleophiles has not been adequately studied. This article is a continuation of our research in ferrocenylalkylation of sulfur-containing heterocyclic compounds ${ }^{11}$ and dealing with a study of these reactions regioselectivity. Pyrrolidine-2-thione (2) 2-mercaptopothiazoline (3), and imidazolidin-2-thione (4) were chosen as ambident nucleophiles since they are pharmacophore groups and have a wide range of biological activity. ${ }^{12-14}$ The choice of heterocycles is not accidental, because they are part of many biologically active substances of natural origin and pharmaceuticals. ${ }^{15-19}$

\section{Results and Discussion}

The development of new efficient synthetic approaches towards ferrocene-containing heterocycles for the design of new biologically active compounds is still an ongoing challenge to organic chemists. Introduction of ferrocenylalkyl moiety into different structures can be achieved by ferrocenylalkylation; however, regioselectivity of this reaction is still poorly studied. Earlier we have described phase-transfer catalyzed alkylation of aromatic mercaptoheterocycles with ferrocenyl carbinols. ${ }^{21}$ It has been shown that the reaction is regioselective and proceeds on nitrogen atom of heterocycle. This was somewhat surprising, because sulfur is considered to be the best nucleophile relative to nitrogen, and in addition, alkylation at the nitrogen atom in 2-mercaptobenzoxazole and 2-mercaptobenzothiazole results in a loss of aromaticity in the reaction products. Furthermore, quantum chemical calculations show that $\mathrm{N}$-isomers are more thermodynamically stable than the corresponding S-isomers. ${ }^{11}$ Moreover, it was shown that under solvation conditions substitution at the nitrogen atom is always more favorable than substitution at the sulfur atom in aromatic heterocycles. This article is devoted to the study of ferrocenylalkylation regioselectivity of such non-aromatic heterocycles as pyrrolidin-2-thione, 2-mercaptothiazoline and imidazolidin-2-thione under the conditions of phase-transfer catalysis.

Reactions of $\alpha$-hydroxyalkylferrocenes with different nucleophilic compounds proceed most efficiently in two-component system, such as liquid-liquid. The reaction of equimolar amounts of $\alpha$-ferrocenylcarbinoles and heterocycles is carried out with vigorous stirring at room temperature. Two-phase system consists of an organic solvent $\left(\mathrm{CH}_{2} \mathrm{Cl}_{2}\right)$ and aqueous solution (45\%) of a tetrafluoroboronic acid. Under these conditions reactions proceed predominantly at the organic inorganic interface. Methylene dichloride contains the 
hydrophobic ferrocene compounds (initial and/or reaction product), and the aqueous phase contains acid. The polar hydroxy group of the organometallic carbinol is exposed to the aqueous phase and is thus available for protonation and formation under mild conditions of thermodynamically stable ferrocenylcarbocation, while the other part of ferrocene compound remains in the organic phase and is not subjected to protonation and oxidation. Therefore, the efficient interaction of the ferrocenyl carbocation with the corresponding nucleophilic reagent may occur, and other reactions (rearrangement, dimerisation, polymerisation, formation of ethers, etc.) are practically excluded.
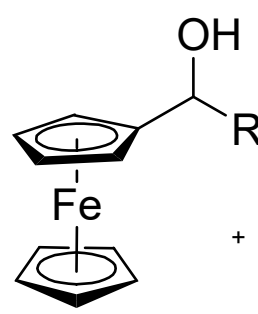

$1(\mathrm{a}-\mathrm{d}, \mathrm{f})$

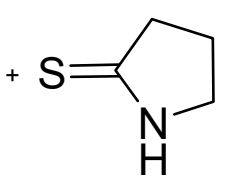

2

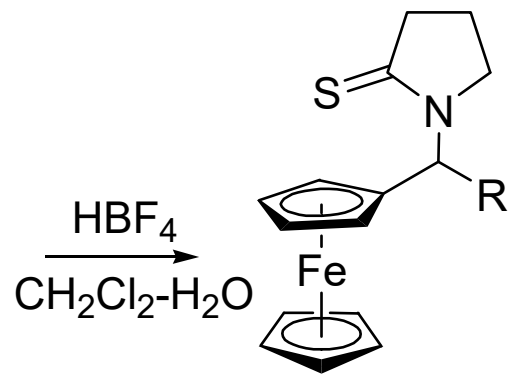

$5(a-d, f)$

$$
\mathrm{R}=\mathrm{H}(\mathrm{a}), \mathrm{CH}_{3}(\mathrm{~b}), \mathrm{C}_{2} \mathrm{H}_{5}(\mathrm{c}), \mathrm{C}_{3} \mathrm{H}_{7}(\mathrm{~d}), \mathrm{C}_{6} \mathrm{H}_{5}(\mathrm{f})
$$

Scheme 1. Ferrocenylalkylation of pyrrolidine-2-thione 2.

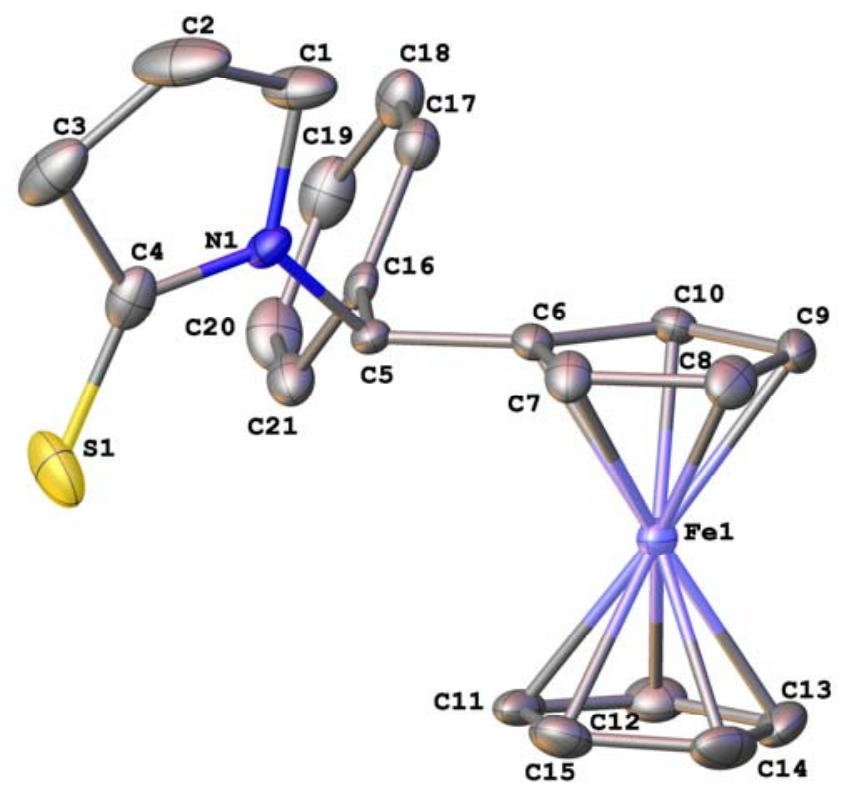

Figure 1. General view of molecule of 1-(ferrocenyl(phenyl)methyl)pyrrolidine-2-thione $\mathbf{5 f}$ presented in anisotropic displacement ellipsoids at 50\% probability. Selected mean bond length and angles ( $\left(\AA\right.$ and $\left.{ }^{\circ}\right)$ : C5-C6 1.515(2), C5-C16 1.516(3), N1-C5 1.472(3), N1-C1 1.340(4), S1-C4 1.661(3), C3-C4 1.504(3), C6-C5N1 110.48(18), N1-C4-S1 126.5(2). 


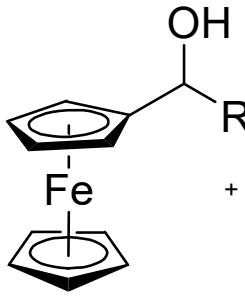

$1(\mathrm{a}-\mathrm{c}, \mathrm{e}, \mathrm{f})$<smiles>[R][SH]=C1NCCS1</smiles>

3

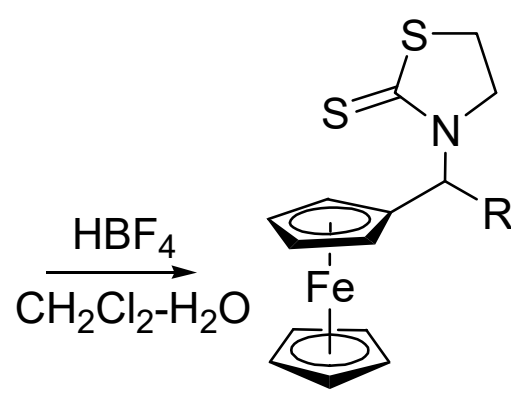

$6(a-c, e, f)$

$$
\mathrm{R}=\mathrm{H}(\mathrm{a}), \mathrm{CH}_{3}(\mathrm{~b}), \mathrm{C}_{2} \mathrm{H}_{5}(\mathrm{c}), i-\mathrm{C}_{3} \mathrm{H}_{7}(\mathrm{e}), \mathrm{C}_{6} \mathrm{H}_{5} \text { (f) }
$$

Scheme 2. Ferrocenylalkylation of 2-mercaptothiazoline 3.

It is found that all reactions proceed regioselectively at the heterocyclic nitrogen atom. Ferrocenylalkylation reaction in acidic media using ferrocenylcarbinols as alkylating agents started with formation of thermodynamically stable $\alpha$-ferrocenium carbocation, which is relatively hard Lewis acid, efficiently react with somewhat harder basic center, in our case, with the nitrogen atom. Furthermore in our previous paper ${ }^{11}$ quantum chemical calculations showed that $\mathrm{N}$-isomers are more thermodynamically stable than the corresponding S-isomers. Structures of the reaction products $(\mathbf{5} \mathbf{a}-\mathbf{d}, \mathbf{f}, \mathbf{6}$ a-c,e,f) $($ Scheme 1,2) were established by ${ }^{1} \mathrm{H}-{ }^{13} \mathrm{C}$ heteronuclear correlation experiments. Thus, HMBC NMR spectra of compounds exhibit characteristic correlations of the bridging alkyl group protons of the ferrocenylalkyl moieties with the $C$ atoms of the $C=S$ group (at about $\delta 180$ ) and with the $C(5)$ atoms of heterocycles. And, vice versa, there is correlation between $\mathrm{C}(5) \mathrm{H}$ and linkage carbon atom. This is evidence in support of the substitution at the heterocyclic rings nitrogen.

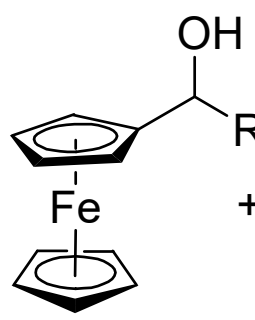

$1(\mathrm{a}-\mathrm{c}, \mathrm{e}, \mathrm{f})$<smiles>[R][SH+]=C1NCCN1</smiles>

4

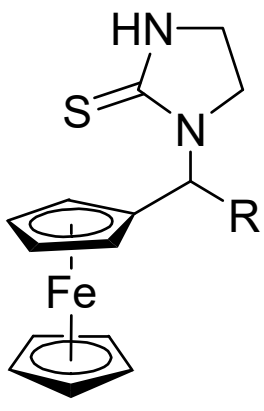

$7(a-c, e, f)$

$$
\mathrm{R}=\mathrm{H}(\mathrm{a}), \mathrm{CH}_{3}(\mathrm{~b}), \mathrm{C}_{2} \mathrm{H}_{5}(\mathrm{c}), i-\mathrm{C}_{3} \mathrm{H}_{7}(\mathrm{e}), \mathrm{C}_{6} \mathrm{H}_{5} \text { (f) }
$$

Scheme 3. Ferrocenylalkylation of imidazolidin-2-thione 4.

Ferrocenylalkylation of imidazole under the acid catalysis conditions in a two-phase aqueous-organic medium is impossible because of its high basicity, as a result of which the protonated imidazole salt is the single product of the reaction. Consequently, a method was developed for obtaining ferrocenylalkyl imidazoles based on the interaction of ferrocene alcohols with carbonyl- or thionyl-diimidazoles. ${ }^{21}$ In contrast to 
imidazole and benzimidazole mercaptoimidazole and mercaptobenzimidazole are less basic so its ferrocenylalkylation possible under acid catalysis. In our early works ferrocenylalkylation was carried out in acetone using $\mathrm{CF}_{3} \mathrm{COOH}$ as a catalyst, ${ }^{22}$ in our work we carried out the alkylation in a two-phase system. ${ }^{11}$ Structures of the reaction products $(\mathbf{7} \mathrm{a}-\mathrm{c}, \mathrm{e}, \mathbf{f})$ (Scheme $\mathbf{3})$ were established by ${ }^{1} \mathrm{H}-{ }^{13} \mathrm{C}$ heteronuclear correlation experiments, moreover structures of the two compounds were established by the X-ray diffraction method. Xray crystallographic studies of $\mathbf{7 a}$ and $\mathbf{7 f}$ (Fig. $\mathbf{2}$ and $\mathbf{3}$ ) show the typical for ferrocene derivatives bond lengths and angles. Apparently, the replacement of one hydrogen atoms bonded to tertiary carbon atom by $i-\operatorname{Pr}$ or $\mathrm{Ph}$ group lead to elongation of the distance between substituted thione group with ferrocenyl fragment. In crystal of 7a the presence of $\mathrm{NH}$ group lead to formation of centrosymmetric dimers assembled via N-H...S bonds (Fig. S8). In crystal packing of $\mathbf{7} \mathbf{d}$ due to $i$-Pr group molecules are assembled to infinite chains along to $a$ side of unit cell (Fig. S9).

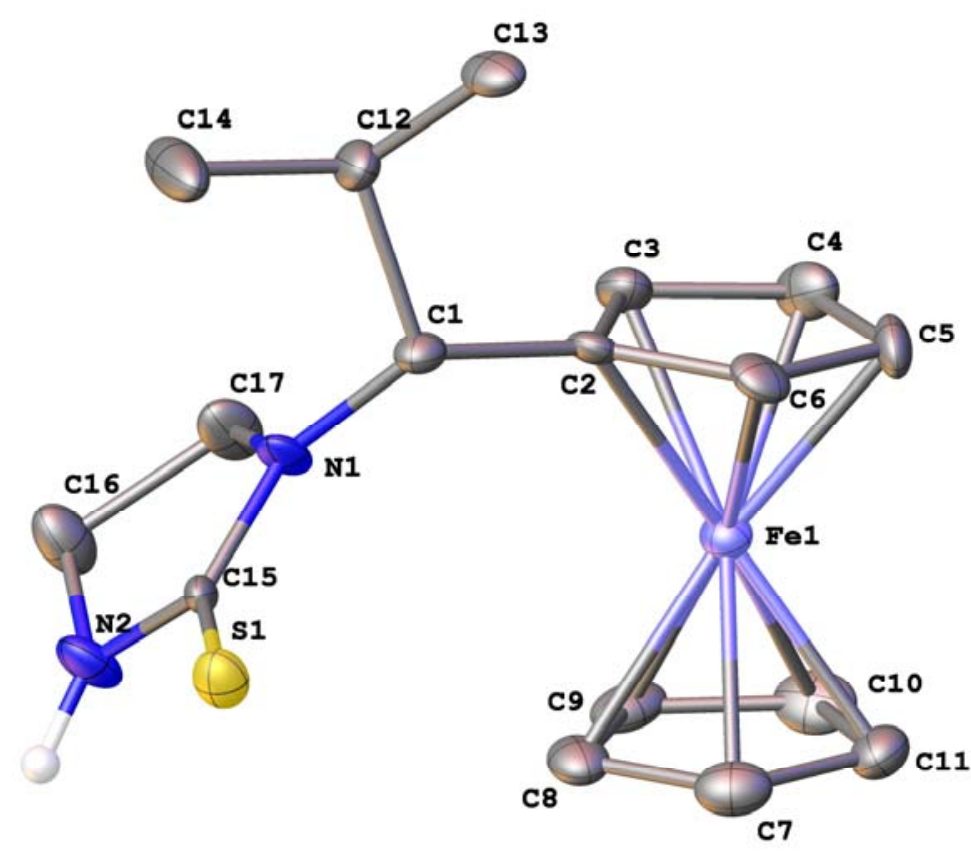

Figure 2. General view of molecule 7e presented in anisotropic displacement ellipsoids at $50 \%$ probability. Selected mean bond length and angles ( $\AA$ and ${ }^{\circ}$ ): C1-C2 1.508(12), C1-C12 1.534(11), N1-C1 1.464(10), N1-C15 1.344(10), 1.358(10), C15-S1 1.675(7), C2-C1-C12 113.3(6), N1-C15-S1 128.1(6), N1-C15-N2 108.6(6). 


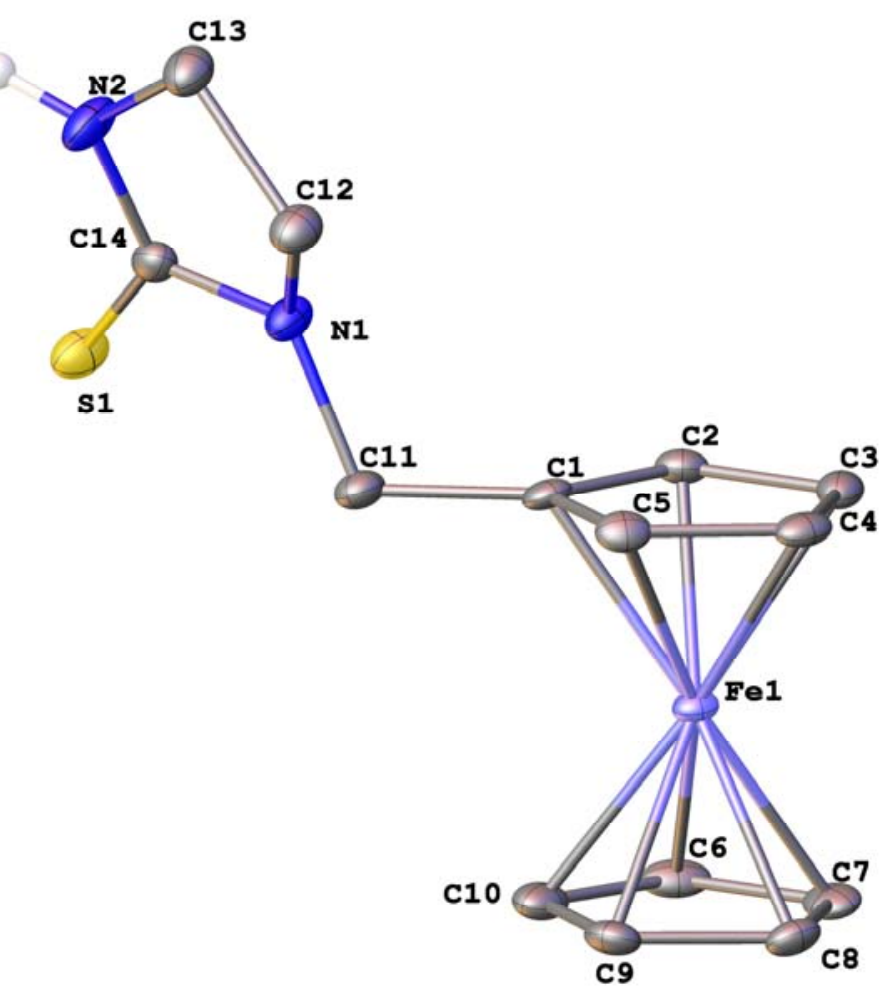

Figure 3. General view of molecule 7a presented in anisotropic displacement ellipsoids at $50 \%$ probability. Selected bond length and angles ( $\AA$ and ${ }^{\circ}$ ): C1-C11 1.488(3), C11-N1 1.460(3), N1-C14 1.350(3), C14-N2 1.335(3), C14-S1 1.683(2), C1-C11-N1 112.21(19), N1-C14-S1 125.51(19), N1-C14-N2 109.0(2)

\section{Conclusions}

Ferrocene containing derivatives of 2-mercaptopothiazoline $\mathbf{5}$ a-d,f, pyrrolidine-2-thione $\mathbf{6}$ a-c,e,f and imidazolidin-2-thione $\mathbf{7}$ a-c,e,f were prepared via the reaction of the $\alpha$-(hydroxy)alkyl ferrocenes with corresponding heterocycles in acidic media. Under these conditions, the alkylation takes place on the nitrogen of heterocycles and not by sulfur moiety.

\section{Experimental Section}

General. Solvents were dried following the standard procedures and distilled under argon prior to use. El mass spectra were taken on a FINNIGAN POLARIS Q spectrometer at $70 \mathrm{eV}$ and the temperature of the ion chamber $250{ }^{\circ} \mathrm{C} .{ }^{1} \mathrm{H}$ and ${ }^{13} \mathrm{C}$ NMR spectra were obtained on a Bruker Avance 400 spectrometer (at 400 and $100 \mathrm{MHz}$, respectively) in $\mathrm{CDCl}_{3}$ at $30{ }^{\circ} \mathrm{C}$. The chemical shifts are given in the $\delta$ scale relative to the residual solvent signal. All signals in the NMR spectra were attributed using gradient HSQC and HMBC heteronuclear correlation techniques. Melting points were determined with a Stuart SMP30 apparatus (Bibby Scientific). Elemental analysis data were obtained in the Laboratory of microanalysis of A.N. Nesmeyanov Institute of Organoelement Compounds (INEOS RAS). Commercially available pyrrolidine-2-thione (2) 2mercaptopothiazoline (3), and imidazolidin-2-thione (4) (Acros Organics) were used as purchased. Ferrocenylmethanol (1a) was synthesized from trimethylferrocenylmethylammonium iodide by the known procedure. ${ }^{23}$ Ferrocenyl carbinols $(\mathbf{1} \mathbf{b}-\mathbf{e})$ were synthesized from ferrocene by the Friedel-Crafts acylation 
with the corresponding acid chlorides and subsequent reduction with lithium aluminum hydride in diethyl ether or THF. ${ }^{24}$

General Procedure. To a mixture of $1.0 \mathrm{mmol}$ of ferrocenylcarbinol and $1.0 \mathrm{mmol}$ of the corresponding heterocycle in $1.0 \mathrm{ml}$ of methylene dichloride, $0.18 \mathrm{ml}$ of $45 \%$ aqueous solution of fluoroboric acid was added under vigorous stirring. The agitation was continued for $5 \mathrm{~min}$ then $\mathrm{Et}_{2} \mathrm{O}(15 \mathrm{ml})$, the same amount of cold water, and 5-10 mg of ascorbic acid were added to the reaction flask. After vigorous shaking of the mixture the organic solution was separated, washed with cold water $(3 \times 15 \mathrm{ml})$, the solvent was removed and the residue was dried over $\mathrm{CaCl}_{2}$. All types of products (pyrrolidine as well as imidazolidine and thiazolidine derivatives) were equally purified, namely by column chromatography (silica, eluent hexane EtOAc 3:1), and solids obtained after chromatography were crystalized from ethanol.

1-(1-Ferrocenylmethyl)pyrrolidine-2-thione (5a). (85\%); orange powder; mp $104.8-105.6{ }^{\circ} \mathrm{C} .{ }^{1} \mathrm{H}$ NMR (400 $\left.\mathrm{MHz}, \mathrm{CDCl}_{3}\right) \delta(\mathrm{ppm}): 2.02\left(\mathrm{~m}, 2 \mathrm{H}, \mathrm{CH}_{2}\right), 2.61\left(\mathrm{~m}, 2 \mathrm{H}, \mathrm{CH}_{2}\right), 3.90\left(\mathrm{~m}, 2 \mathrm{H}, \mathrm{CH}_{2}\right), 4.12(\mathrm{~s}, 2 \mathrm{H}, \mathrm{Fc}), 4.14\left(\mathrm{~s}, 2 \mathrm{H}, \mathrm{CH}_{2}\right)$, $4.13(\mathrm{~s}, 5 \mathrm{H}, \mathrm{Fc}), 4.25$ (s, 2H, Fc). ${ }^{13} \mathrm{C} \mathrm{NMR}\left(100 \mathrm{MHz}, \mathrm{CDCl}_{3}\right) \delta(\mathrm{ppm}): 19.8\left(\mathrm{CH}_{2}\right), 45.2\left(\mathrm{CH}_{2}\right), 49.3\left(\mathrm{CH}_{2}\right), 52.1$ $(\mathrm{CH}), 66.0\left(\mathrm{C}_{5} \mathrm{H}_{4}\right), 67.6\left(\mathrm{C}_{5} \mathrm{H}_{4}\right), 68.8\left(\mathrm{C}_{5} \mathrm{H}_{4}\right), 68.9\left(\mathrm{C}_{5} \mathrm{H}_{4}\right), 69.1\left(\mathrm{C}_{5} \mathrm{H}_{5}\right), 86.9$ (ipso- $\left.\mathrm{C}_{5} \mathrm{H}_{4}\right), 200.1$ (C=S) Calc. for $\mathrm{C}_{15} \mathrm{H}_{17} \mathrm{FeNS}: 61.22 ; \mathrm{H}, 5.74 ; \mathrm{N}, 4.69 ; \mathrm{Fe}, 18.67 ; \mathrm{S}, 10.72$. Found: $\mathrm{C}, 60.21 ; \mathrm{H}, 5.73 ; \mathrm{Fe}, 18.66 ; \mathrm{N}, 4.68 ; \mathrm{S}, 10.72$. $\mathrm{El} / \mathrm{MS}, \mathrm{m} / z$ (RI\%): $299[\mathrm{M}]^{+}(46)$.

1-(1-Ferrocenylethyl)pyrrolidine-2-thione (5b). (75\%); yellow powder, mp 88.1 ${ }^{\circ} \mathrm{C}$. El/MS, m/z (RI\%): 313 [M] (69). ${ }^{1} \mathrm{H}$ NMR (400 MHz, $\left.\mathrm{CDCl}_{3}\right) \delta(\mathrm{ppm}): 1.56\left(\mathrm{~d}, 3 \mathrm{H}, J\right.$ 7.0, $\left.\mathrm{CH}_{3}\right), 1.88\left(\mathrm{~m}, 2 \mathrm{H}, \mathrm{CH}_{2}\right), 2.99\left(\mathrm{~m}, 2 \mathrm{H}, \mathrm{CH}_{2}\right), 3.23(\mathrm{~m}$, $1 \mathrm{H}, \mathrm{CH}), 3.46(\mathrm{~m}, \mathrm{H}, \mathrm{CH}), 4.11(\mathrm{~s}, 1 \mathrm{H}, \mathrm{Fc}), 4.17(\mathrm{~s}, 2 \mathrm{H}, \mathrm{Fc}), 4.21(\mathrm{~s}, 5 \mathrm{H}, \mathrm{Fc}), 4.30(\mathrm{~s}, 1 \mathrm{H}, \mathrm{Fc}), 6.08$ (q, 1H, J 7.1, CH). ${ }^{13} \mathrm{C} \mathrm{NMR}\left(100 \mathrm{MHz}, \mathrm{CDCl}_{3}\right) \delta(\mathrm{ppm}): 15.6\left(\mathrm{CH}_{3}\right), 19.4\left(\mathrm{CH}_{2}\right), 45.2\left(\mathrm{CH}_{2}\right), 49.2\left(\mathrm{CH}_{2}\right), 51.5(\mathrm{CH}), 66.0\left(\mathrm{C}_{5} \mathrm{H}_{4}\right), 67.5$ $\left(\mathrm{C}_{5} \mathrm{H}_{4}\right), 68.6\left(\mathrm{C}_{5} \mathrm{H}_{4}\right), 68.8\left(\mathrm{C}_{5} \mathrm{H}_{4}\right), 68.9\left(\mathrm{C}_{5} \mathrm{H}_{5}\right), 86.7$ (ipso- $\left.\mathrm{C}_{5} \mathrm{H}_{4}\right), 199.2(\mathrm{C}=\mathrm{S})$. Calc. for $\mathrm{C}_{16} \mathrm{H}_{19} \mathrm{FeNS}: \mathrm{C}, 61.35 ; \mathrm{H}$, 6.11; Fe, 17.83; N, 4.47; S, 10.24. Found: C, 61.33; H, 6.12; Fe, 17.82; N, 4.48.

1-(1-Ferrocenylpropyl)pyrrolidine-2-thione (5c). (80\%); yellow oil. El/MS, m/z (RI\%): $327[\mathrm{M}]^{+}(68) .{ }^{1} \mathrm{H}$ NMR $\left(400 \mathrm{MHz}, \mathrm{CDCl}_{3}\right) \delta(\mathrm{ppm}):{ }^{1} \mathrm{H} \mathrm{NMR}\left(500 \mathrm{MHz}, \mathrm{CDCl}_{3}\right) \delta(\mathrm{ppm}) 0.99\left(\mathrm{t}, J 7.4 \mathrm{~Hz}, 3 \mathrm{H}, \mathrm{CH}_{3}\right), 1.85(\mathrm{~m}, 1 \mathrm{H}), 1.94(\mathrm{~m}$, 1H), 2.19 (dqd, J 14.9, 7.5, $4.0 \mathrm{~Hz}, 1 \mathrm{H}$ ), $3.00(\mathrm{~m}, 1 \mathrm{H}$ ), 3.25 (ddd, J 11.1, 8.5, 6.2 Hz, 1H), 3.41 (ddd, J 11.1, 8.4, $6.1 \mathrm{~Hz}, 1 \mathrm{H}), 4.12(\mathrm{~s}, 2 \mathrm{H}, \mathrm{Fc}), 4.15(\mathrm{~s}, 1 \mathrm{H}, \mathrm{Fc}), 4.18(\mathrm{~s}, 5 \mathrm{H}, \mathrm{Fc}), 4.31(\mathrm{~d}, J 1.5 \mathrm{~Hz}, 1 \mathrm{H}), 6.01(\mathrm{dd}, J 11.6,3.9 \mathrm{~Hz}, 1 \mathrm{H}$, $\mathrm{CH}) .{ }^{13} \mathrm{C}$ NMR $\left(126 \mathrm{MHz}, \mathrm{CDCl}_{3}\right) \delta(\mathrm{ppm}): 10.94\left(\mathrm{CH}_{3}\right), 19.62\left(\mathrm{CH}_{2}\right), 24.00\left(\mathrm{CH}_{2}\right), 45.20\left(\mathrm{CH}_{2}\right), 48.62\left(\mathrm{CH}_{2}\right), 56.66$ $(\mathrm{CH}), 66.08\left(\mathrm{C}_{5} \mathrm{H}_{4}\right), 67.29\left(\mathrm{C}_{5} \mathrm{H}_{4}\right), 68.21\left(\mathrm{C}_{5} \mathrm{H}_{4}\right), 68.52\left(\mathrm{C}_{5} \mathrm{H}_{4}\right), 69.01\left(\mathrm{C}_{5} \mathrm{H}_{5}\right), 86.72$ (ipso- $\left.\mathrm{C}_{5} \mathrm{H}_{4}\right), 200.99$ (C=S). Calc. for $\mathrm{C}_{17} \mathrm{H}_{21}$ FeNS: C, 62.39; H, 6.47; Fe, 17.06; N, 4.28; S, 9.80. Found: $\mathrm{C}, 62.37 ; \mathrm{H}, 6.51 ; \mathrm{N}, 4.29 ; \mathrm{Fe}, 17.00$.

1-(1-Ferrocenylbutyl)pyrrolidine-2-thione (5d). (77\%); brown oil. El/MS, $m / z$ (RI\%): $341[\mathrm{M}]^{+}$(67). ${ }^{1} \mathrm{H}$ NMR $\left(400 \mathrm{MHz}, \mathrm{CDCl}_{3}\right) \delta(\mathrm{ppm}): 1.08\left(\mathrm{t}, 3 \mathrm{H}, J \mathrm{~J} .3, \mathrm{CH}_{3}\right), 1.41\left(\mathrm{~m}, 2 \mathrm{H}, \mathrm{CH}_{2}\right), 1.99\left(\mathrm{~m}, 2 \mathrm{H}, \mathrm{CH}_{2}\right), 3.03\left(\mathrm{~m}, 2 \mathrm{H}, \mathrm{CH}_{2}\right), 3.27$ $\left(\mathrm{m}, 2 \mathrm{H}, \mathrm{CH}_{2}\right), 3.45\left(\mathrm{~m}, 2 \mathrm{H}, \mathrm{CH}_{2}, \mathrm{Het}\right), 4.15\left(\mathrm{~s}, 2 \mathrm{H}, 2 \mathrm{CH}, \mathrm{C}_{5} \mathrm{H}_{4}\right), 4.18\left(\mathrm{~s}, \mathrm{H}, \mathrm{CH}, \mathrm{C}_{5} \mathrm{H}_{4}\right), 4.21\left(\mathrm{~s}, 5 \mathrm{H}, \mathrm{C}_{5} \mathrm{H}_{5}\right), 4.36(\mathrm{~s}, 1 \mathrm{H}$, $\left.\mathrm{CH}, \mathrm{C}_{5} \mathrm{H}_{4}\right), 6.13(\mathrm{~m}, 1 \mathrm{H}, \mathrm{CH}) .{ }^{13} \mathrm{C}$ NMR $\left(101 \mathrm{MHz}, \mathrm{CDCl}_{3}\right) \delta 11.1\left(\mathrm{CH}_{3}\right), 14.2\left(\mathrm{CH}_{2}\right), 19.4\left(\mathrm{CH}_{2}\right), 27.5\left(\mathrm{CH}_{2}\right), 33.2$ $\left(\mathrm{CH}_{2}\right), 51.0\left(\mathrm{CH}_{2}\right), 56.4(\mathrm{CH}), 66.1\left(\mathrm{C}_{5} \mathrm{H}_{4}\right), 67.5\left(\mathrm{C}_{5} \mathrm{H}_{4}\right), 68.3\left(\mathrm{C}_{5} \mathrm{H}_{4}\right), 68.8\left(\mathrm{C}_{5} \mathrm{H}_{4}\right), 69.1\left(\mathrm{C}_{5} \mathrm{H}_{5}\right), 86.6\left(\right.$ ipso- $\left.\mathrm{C}_{5} \mathrm{H}_{4}\right), 196.0$ (C=S).Calc. for $\mathrm{C}_{18} \mathrm{H}_{23}$ FeNS: C, 63.35; H, 6.79; N, 4.10; Fe, 16.36; S, 9.39. Found: C, 63.32; H, 6.74; N, 4.12; Fe, 16.33.

1-(Ferrocenyl(phenyl)methyl)pyrrolidine-2-thione (5f). (72\%); orange powder, mp $131.5-132.3^{\circ} \mathrm{C}$. El/MS, $\mathrm{m} / z$ (RI\%): $375[\mathrm{M}]^{+}(27) .{ }^{1} \mathrm{H} \mathrm{NMR}\left(400 \mathrm{MHz}, \mathrm{CDCl}_{3}\right) \delta(\mathrm{ppm}): 1.98\left(\mathrm{~m}, 2 \mathrm{H}, \mathrm{CH}_{2}\right), 3.08\left(\mathrm{~m}, 2 \mathrm{H}, \mathrm{CH}_{2}\right), 3.45(\mathrm{~m}, 1 \mathrm{H}$, $\mathrm{CH}), 3.70(\mathrm{~m}, 1 \mathrm{H}, \mathrm{CH}), 4.18\left(\mathrm{~s}, 5 \mathrm{H}, \mathrm{C}_{5} \mathrm{H}_{5}\right), 4.22\left(\mathrm{~s}, 1 \mathrm{H}, \mathrm{CH}, \mathrm{C}_{5} \mathrm{H}_{4}\right), 4.26\left(\mathrm{~m}, 4 \mathrm{H}, \mathrm{CH}, \mathrm{C}_{5} \mathrm{H}_{4}+\mathrm{CH}\right), 7.37\left(\mathrm{~m}, 5 \mathrm{H}, \mathrm{C}_{6} \mathrm{H}_{5}\right)$. ${ }^{13} \mathrm{C} \mathrm{NMR}\left(100 \mathrm{MHz}, \mathrm{CDCl}_{3}\right) \delta(\mathrm{ppm}): 20.1\left(\mathrm{CH}_{2}\right), 44.9\left(\mathrm{CH}_{2}\right), 51.1\left(\mathrm{CH}_{2}\right), 59.5(\mathrm{CH}), 67.9\left(\mathrm{C}_{5} \mathrm{H}_{4}\right), 68.2\left(\mathrm{C}_{5} \mathrm{H}_{4}\right), 68.5$ $\left(\mathrm{C}_{5} \mathrm{H}_{4}\right), 69.3\left(\mathrm{C}_{5} \mathrm{H}_{5}\right), 85.3$ (ipso- $\left.\mathrm{C}_{5} \mathrm{H}_{4}\right), 127.93\left(\mathrm{C}_{6} \mathrm{H}_{5}\right), 128.40\left(\mathrm{C}_{6} \mathrm{H}_{5}\right), 128.45\left(\mathrm{C}_{6} \mathrm{H}_{5}\right), 138.1$ (ipso- $\left.\mathrm{C}_{6} \mathrm{H}_{5}\right), 200.7(\mathrm{C}=\mathrm{S})$. Calc. for $\mathrm{C}_{21} \mathrm{H}_{21}$ FeNS: C, 67.21; H, 5.64; N, 3.73; Fe, 14.88. S, 8.54. Found: C 67.23; H 4.65; N 3.75; Fe 14.80. 
3-(1-Ferrocenylmethyl)thiazolidine-2-thione (6a). (57\%);orange powder, $\mathrm{mp} 94.0-95.2^{\circ} \mathrm{C} . \mathrm{El} / \mathrm{MS}, \mathrm{m} / \mathrm{z}$ (RI\%): $317\left[\mathrm{M}^{+}(84) .{ }^{1} \mathrm{H}\right.$ NMR $\left(400 \mathrm{MHz}, \mathrm{CDCl}_{3}\right) \delta(\mathrm{ppm}): 3.09\left(\mathrm{~m}, 2 \mathrm{H}, \mathrm{CH}_{2}\right), 3.59(\mathrm{~m}, 1 \mathrm{H}, \mathrm{CH}), 3.79(\mathrm{~m}, 1 \mathrm{H}, \mathrm{CH}), 4.17$ $\left(\mathrm{m}, 3 \mathrm{H}, \mathrm{C}_{5} \mathrm{H}_{4}\right), 4.20(\mathrm{~s}, 5 \mathrm{H}, \mathrm{Fc}), 4.36(\mathrm{~s}, 1 \mathrm{H}, \mathrm{Fc}), 6.09\left(\mathrm{~s}, 2 \mathrm{H}, \mathrm{CH}_{2}\right) .{ }^{13} \mathrm{C} \mathrm{NMR}\left(100 \mathrm{MHz}, \mathrm{CDCl}_{3}\right) \delta(\mathrm{ppm}): 33.2\left(\mathrm{CH}_{2}\right)$, $51.0\left(\mathrm{CH}_{2}\right), 56.4\left(\mathrm{CH}_{2}\right), 66.1\left(\mathrm{C}_{5} \mathrm{H}_{4}\right), 67.5\left(\mathrm{C}_{5} \mathrm{H}_{4}\right), 68.3\left(\mathrm{C}_{5} \mathrm{H}_{4}\right), 68.8\left(\mathrm{C}_{5} \mathrm{H}_{4}\right), 69.1\left(\mathrm{C}_{5} \mathrm{H}_{4}\right), 86.5\left(\right.$ ipso- $\left.\mathrm{C}_{5} \mathrm{H}_{4}\right), 195.9$ (C=S). Calc. for $\mathrm{C}_{14} \mathrm{H}_{15} \mathrm{FeNS}_{2}$ : C, 53.00; H, $4.77 \mathrm{~N}, 4.42 ; \mathrm{Fe}, 17.60 ; \mathrm{S}, 20.21$. Found: C, 53.04; H, 4.81; Fe, 17.63.

3-(1-Ferrocenylethyl)thiazolidine-2-thione (6b). (61\%); yellow powder, $\mathrm{mp} 123.3-124^{\circ} \mathrm{C}$. El/MS, $\mathrm{m} / \mathrm{z}$ (RI\%): $331[\mathrm{M}]^{+}(73) .{ }^{1} \mathrm{H}$ NMR $\left(400 \mathrm{MHz}, \mathrm{CDCl}_{3}\right) \delta(\mathrm{ppm}): 1.61\left(\mathrm{~d}, 3 \mathrm{H}, J 7.0, \mathrm{CH}_{3}\right), 3.11\left(\mathrm{~m}, 2 \mathrm{H}, \mathrm{CH}_{2}\right), 3.61(\mathrm{~m}, 1 \mathrm{H}, \mathrm{CH})$, $3.83(\mathrm{~m}, 1 \mathrm{H}, \mathrm{CH}), 4.23(\mathrm{~m}, 8 \mathrm{H}, \mathrm{Fc}), 4.36(\mathrm{~s}, 1 \mathrm{H}, \mathrm{Fc}), 6.11(\mathrm{~m}, 1 \mathrm{H}, \mathrm{CH}) .{ }^{13} \mathrm{C} \mathrm{NMR}\left(100 \mathrm{MHz}, \mathrm{CDCl}_{3}\right) \delta(\mathrm{ppm}): 15.4$ $\left(\mathrm{CH}_{3}\right), 29.1\left(\mathrm{CH}_{2}\right), 35.2\left(\mathrm{CH}_{2}\right), 51.5(\mathrm{CH}), 66.3\left(\mathrm{C}_{5} \mathrm{H}_{4}\right), 67.6\left(\mathrm{C}_{5} \mathrm{H}_{4}\right), 68.3\left(\mathrm{C}_{5} \mathrm{H}_{4}\right), 68.8\left(\mathrm{C}_{5} \mathrm{H}_{4}\right), 68.9\left(\mathrm{C}_{5} \mathrm{H}_{5}\right), 86.4$ (ipso$\mathrm{C}_{5} \mathrm{H}_{4}$ ), 195.2 (C=S).Anal.: $\mathrm{C}, 54.37 ; \mathrm{H}, 5.18 ; \mathrm{N}, 4.21 ; \mathrm{Fe}, 16.90 . \mathrm{C}_{15} \mathrm{H}_{17} \mathrm{FeNS}_{2}: \mathrm{C}, 54.38 ; \mathrm{H}, 5.17 ; \mathrm{N}, 4.23 ; \mathrm{Fe}, 16.86$. 3-(1-Ferrocenylpropyl)thiazolidine-2-thione (6c). (47\%); orange oil. El/MS, $m / z$ (RI\%): $345\left[\mathrm{M}^{+}{ }^{+}(45) .{ }^{1} \mathrm{H}\right.$ NMR $\left(400 \mathrm{MHz}, \mathrm{CDCl}_{3}\right) \delta(\mathrm{ppm}): 1.06\left(\mathrm{t}, 3 \mathrm{H}, J \mathrm{~J} .4, \mathrm{CH}_{3}\right), 1.86(\mathrm{~m}, 1 \mathrm{H}, \mathrm{CH}), 2.23(\mathrm{~m}, 1 \mathrm{H}, \mathrm{CH}), 3.11(\mathrm{~m}, 1 \mathrm{H}, \mathrm{CH}), 3.19(\mathrm{~m}$, $1 \mathrm{H}, \mathrm{CH}), 3.61(\mathrm{~m}, 1 \mathrm{H}, \mathrm{CH}), 3.80(\mathrm{~m}, 1 \mathrm{H}, \mathrm{CH}), 4.17\left(\mathrm{~s}, 3 \mathrm{H}, \mathrm{C}_{5} \mathrm{H}_{4}\right), 4.21\left(\mathrm{~s}, 5 \mathrm{H}, \mathrm{C}_{5} \mathrm{H}_{5}\right), 4.36\left(\mathrm{~s}, 1 \mathrm{H}, \mathrm{C}_{5} \mathrm{H}_{4}\right), 6.02(\mathrm{~m}, 1 \mathrm{H}$, $\mathrm{CH}) .{ }^{13} \mathrm{C} \mathrm{NMR}\left(100 \mathrm{MHz}, \mathrm{CDCl}_{3}\right) \delta(\mathrm{ppm}): 16.2\left(\mathrm{CH}_{3}\right), 27.5\left(\mathrm{CH}_{2}\right), 33.2\left(\mathrm{CH}_{2}\right), 51.0\left(\mathrm{CH}_{2}\right), 57.5(\mathrm{CH}), 66.2\left(\mathrm{C}_{5} \mathrm{H}_{4}\right)$, 67. $3\left(\mathrm{C}_{5} \mathrm{H}_{4}\right), 68.2\left(\mathrm{C}_{5} \mathrm{H}_{4}\right), 68.7\left(\mathrm{C}_{5} \mathrm{H}_{4}\right), 69.2\left(\mathrm{C}_{5} \mathrm{H}_{4}\right), 86.7$ (ipso- $\left.\mathrm{C}_{5} \mathrm{H}_{4}\right), 195.8(\mathrm{C}=\mathrm{S})$. Calc. for $\mathrm{C}_{16} \mathrm{H}_{19} \mathrm{FeNS}_{2}: \mathrm{C}, 55.65$; $\mathrm{H}, 5.55 ; \mathrm{N}, 4.06$; Fe, 16.17; S, 18.57. Found: C, 55.68; H, 5.57; N, 4.05; Fe, 16.22.

3-(1-Ferrocenyl-2methylpropyl)thiazolidine-2-thione (6e). (65\%); yellow powder, $\mathrm{mp} 127-128^{\circ} \mathrm{C}$. El/MS, $\mathrm{m} / \mathrm{z}$ (RI\%): $359[\mathrm{M}]^{+}(85) .{ }^{1} \mathrm{H}$ NMR (400 MHz, $\left.\mathrm{CDCl}_{3}\right) \delta(\mathrm{ppm}): 0.97$ (d, 3H, J 6.6, $\mathrm{CH}_{3}$ ), 1.11 (d, 3H, J 6.6, $\left.\mathrm{CH}_{3}\right), 3.23$ $\left(\mathrm{m}, 2 \mathrm{H}, \mathrm{CH}_{2}\right), 3.95\left(\mathrm{~m}, 2 \mathrm{H}, \mathrm{CH}_{2}\right), 4.10(\mathrm{~s}, 1 \mathrm{H}, \mathrm{Fc}), 4.19(\mathrm{~s}, 8 \mathrm{H}, \mathrm{Fc}), 5.61(\mathrm{~d}, 1 \mathrm{H}, \mathrm{CH}) .{ }^{13} \mathrm{C} \mathrm{NMR}\left(100 \mathrm{MHz}, \mathrm{CDCl}_{3}\right) \delta$ (ppm): $20.1\left(\mathrm{CH}_{3}\right), 21.2\left(\mathrm{CH}_{3}\right), 27.6(\mathrm{Het}), 31.8$ (Het), $52.4(\mathrm{CH}), 62.7\left(\mathrm{C}_{5} \mathrm{H}_{4}\right), 66.3\left(\mathrm{C}_{5} \mathrm{H}_{4}\right), 66.8\left(\mathrm{C}_{5} \mathrm{H}_{4}\right), 68.5\left(\mathrm{C}_{5} \mathrm{H}_{4}\right)$, $69.2\left(\mathrm{C}_{5} \mathrm{H}_{4}\right), 87.2\left(\right.$ ipso- $\left.\mathrm{C}_{5} \mathrm{H}_{4}\right)$, 195. 7 (C=S). Calc. for $\mathrm{C}_{17} \mathrm{H}_{21} \mathrm{FeNS}_{2}$ : C, 56.82; H, 5.89; N, 3.90; Fe, 15.54; S, 17.84. Found: $\mathrm{C}, 56.83 ; \mathrm{H}, 5.88 ; \mathrm{N}, 3.92 ; \mathrm{Fe}, 15.55$.

3-(1-Ferrocenyl(methyl)phenyl)thyazolidine-2(3H)-thione (6f). (69\%); orange crystals, mp $141.1-143^{\circ} \mathrm{C}$. El/MS, m/z (RI\%): $393[\mathrm{M}]^{+}(36) .{ }^{1} \mathrm{H}$ NMR $\left(400 \mathrm{MHz}, \mathrm{CDCl}_{3}\right) \delta(\mathrm{ppm}): 3.12(\mathrm{~m}, 1 \mathrm{H}, \mathrm{CH}), 3.18(\mathrm{~m}, 1 \mathrm{H}, \mathrm{CH}), 3.73$ $(\mathrm{m}, 1 \mathrm{H}, \mathrm{CH}), 4.04(\mathrm{~m}, 1 \mathrm{H}, \mathrm{CH}), 4.18(\mathrm{~s}, 1 \mathrm{H}, \mathrm{Fc}), 4.20(\mathrm{~s}, 5 \mathrm{H}, \mathrm{Fc}), 4.24(\mathrm{~s}, 1 \mathrm{H}, \mathrm{Fc}), 4.26(\mathrm{~s}, 1 \mathrm{H}, \mathrm{Fc}), 4.28(\mathrm{~s}, 1 \mathrm{H}, \mathrm{Fc})$, $7.36(\mathrm{~m}, 5 \mathrm{H}, \mathrm{Ph}) .{ }^{13} \mathrm{C} \mathrm{NMR}\left(100 \mathrm{MHz}, \mathrm{CDCl}_{3}\right) \delta(\mathrm{ppm}): 53.1\left(\mathrm{CH}_{2}\right), 60.5(\mathrm{CH}), 67.9\left(\mathrm{C}_{5} \mathrm{H}_{4}\right), 68.1\left(\mathrm{C}_{5} \mathrm{H}_{4}\right), 68.6\left(\mathrm{C}_{5} \mathrm{H}_{4}\right)$, $69.4\left(\mathrm{C}_{5} \mathrm{H}_{4}\right), 69.4\left(\mathrm{C}_{5} \mathrm{H}_{5}\right), 85.3\left(\right.$ ipso- $\left.\mathrm{C}_{5} \mathrm{H}_{4}\right), 128.1\left(\mathrm{C}_{6} \mathrm{H}_{5}\right), 128.3\left(\mathrm{C}_{6} \mathrm{H}_{5}\right), 128.6\left(\mathrm{C}_{6} \mathrm{H}_{5}\right), 138.1$ (ipso- $\left.\mathrm{C}_{6} \mathrm{H}_{5}\right), 196.1$ (C=S). Calc. for $\mathrm{C}_{20} \mathrm{H}_{19} \mathrm{FeNS}_{2}$ : C, 61.07; H, 4.87; N, 3.56; Fe, 14.20; S, 17.84. Found: C, 61.10; H, 4.85; N, 3.52; Fe, 14.28.

4,5-Dihydro-1-(1-ferrocenylmethyl)-1H-imidazole-2-thiol (7a). (69\%); orange crystals, mp $184^{\circ} \mathrm{C}$. El/MS, $\mathrm{m} / \mathrm{z}$ (RI\%): $300[\mathrm{M}]^{+}(32) .{ }^{1} \mathrm{H}$ NMR $\left(400 \mathrm{MHz}, \mathrm{CDCl}_{3}\right) \delta 3.48(\mathrm{~m}, 4 \mathrm{H}), 4.13(\mathrm{~s}, 2 \mathrm{H}, \mathrm{Fc}), 4.18(\mathrm{~s}, 5 \mathrm{H}, \mathrm{Fc}), 4.26(\mathrm{~s}, 2 \mathrm{H}, \mathrm{Fc})$, $4.54\left(\mathrm{~s}, 2 \mathrm{H}, \mathrm{CH}_{2}\right), 5.92(\mathrm{~s}, 1 \mathrm{H}, \mathrm{NH}), .{ }^{13} \mathrm{C} \mathrm{NMR}\left(101 \mathrm{MHz}, \mathrm{CDCl}_{3}\right) \delta 41.2\left(\mathrm{CH}_{2}\right), 46.5\left(\mathrm{CH}_{2}\right), 47.8\left(\mathrm{CH}_{2}\right), 68.3\left(\mathrm{C}_{5} \mathrm{H}_{4}\right)$, $68.4\left(\mathrm{C}_{5} \mathrm{H}_{4}\right), 68.7\left(\mathrm{C}_{5} \mathrm{H}_{4}\right), 69.5\left(\mathrm{C}_{5} \mathrm{H}_{5}\right), 81.7$ (ipso- $\left.\mathrm{C}_{5} \mathrm{H}_{4}\right), 182.4$ (C=S).Calc. for $\mathrm{C}_{14} \mathrm{H}_{16} \mathrm{FeN}_{2} \mathrm{~S}: \mathrm{C}, 56.01 ; \mathrm{H}, 5.37 ; \mathrm{N}$, 9.33; Fe, 18.60; S, 10.68. Found: C, 56.00; H, 5.40; N, 9.35; Fe, 18.50.

4,5-Dihydro-1-(1-ferrocenylethyl)-1H-imidazole-2-thiol (7b). (72\%); orange crystals, $\mathrm{mp} 160^{\circ} \mathrm{C}$. El/MS, $\mathrm{m} / \mathrm{z}$ (RI\%): $314[\mathrm{M}]^{+}$(48). ${ }^{1} \mathrm{H} \mathrm{NMR}\left(400 \mathrm{MHz}, \mathrm{CDCl}_{3}\right) \delta 5.99$ (s, 1H, NH), 5.72 (q, J 7.0, 1H, CH), 4.36 (s, 1H, Fc), 4.15 $(\mathrm{m}, 8 \mathrm{H}, \mathrm{Fc}), 3.52-3.35\left(\mathrm{~m}, 3 \mathrm{H}, \mathrm{CH}_{2}+\mathrm{CH}\right), 3.25-3.12\left(\mathrm{~m}, 1 \mathrm{H}, \mathrm{CH}_{2}\right), 1.52\left(\mathrm{~d}, J 7.0,3 \mathrm{H}, \mathrm{CH}_{3}\right) .{ }^{13} \mathrm{C} \mathrm{NMR}(101 \mathrm{MHz}$, $\left.\mathrm{CDCl}_{3}\right)$ \& $16.2\left(\mathrm{CH}_{3}\right), 41.4\left(\mathrm{CH}_{2}\right), 43.4\left(\mathrm{CH}_{2}\right), 50.5(\mathrm{CH}), 66.0\left(\mathrm{C}_{5} \mathrm{H}_{4}\right), 67.4\left(\mathrm{C}_{5} \mathrm{H}_{4}\right), 68.6\left(\mathrm{C}_{5} \mathrm{H}_{4}\right), 68.7\left(\mathrm{C}_{5} \mathrm{H}_{4}\right), 68.9$ $\left(\mathrm{C}_{5} \mathrm{H}_{5}\right), 87.4$ (ipso- $\left.\mathrm{C}_{5} \mathrm{H}_{4}\right), 182.1$ (C=S). Calc. for $\mathrm{C}_{15} \mathrm{H}_{18} \mathrm{FeN}_{2} \mathrm{~S}: \mathrm{C}, 57.34 ; \mathrm{H}, 5.77 ; \mathrm{N}, 8.92 ; \mathrm{Fe}, 17.77 ; \mathrm{S}, 10.20$. Found: C, 57.32; H, 5.80; N, 8.90; Fe, 17.80.

4,5-Dihydro-1-(1-ferrocenylpropyl)-1H-imidazole-2-thiol (7c). (66\%); yellow oil. El/MS, $\mathrm{m} / \mathrm{z}$ (RI\%): 328 [M] $]^{+}$ (48). ${ }^{1} \mathrm{H}$ NMR (400 MHz, CDCl 3 ) $\delta 5.99(\mathrm{~s}, 1 \mathrm{H}, \mathrm{NH}), 5.72(\mathrm{q}, \mathrm{J} 7.0,1 \mathrm{H}, \mathrm{CH}), 4.36(\mathrm{~s}, 1 \mathrm{H}, \mathrm{Fc}), 4.15(\mathrm{~m}, 8 \mathrm{H}, \mathrm{Fc}), 3.52$ - $3.35\left(\mathrm{~m}, 3 \mathrm{H}, \mathrm{CH}_{2}+\mathrm{CH}\right), 3.25-3.12\left(\mathrm{~m}, 1 \mathrm{H}, \mathrm{CH}_{2}\right), 1.52\left(\mathrm{~d}, J 7.0,3 \mathrm{H}, \mathrm{CH}_{3}\right) .{ }^{13} \mathrm{C} \mathrm{NMR}\left(101 \mathrm{MHz}, \mathrm{CDCl}_{3}\right) \delta 10.2$ $\left(\mathrm{CH}_{3}\right), 16.3\left(\mathrm{CH}_{2}\right), 27.5\left(\mathrm{CH}_{2}\right), 41.2\left(\mathrm{CH}_{2}\right), 43.5\left(\mathrm{CH}_{2}\right), 52.5(\mathrm{CH}), 66.2\left(\mathrm{C}_{5} \mathrm{H}_{4}\right), 67.3\left(\mathrm{C}_{5} \mathrm{H}_{4}\right), 68.2\left(\mathrm{C}_{5} \mathrm{H}_{4}\right), 68.7\left(\mathrm{C}_{5} \mathrm{H}_{4}\right)$, 
$69.2\left(\mathrm{C}_{5} \mathrm{H}_{4}\right), 86.7$ (ipso- $\mathrm{C}_{5} \mathrm{H}_{4}$ ), 182.6 (C=S). Calc. for $\mathrm{C}_{16} \mathrm{H}_{20} \mathrm{FeNS}_{2}$ : C, 58.54; H, 6.14; N, 8.53; Fe, 17.01; S, 9.77. Found: $\mathrm{C}, 58.53 ; \mathrm{H}, 6.10 ; \mathrm{N}, 8.50 ; \mathrm{Fe}, 16.96$.

4,5-Dihydro-1-(1-ferrocenyl-2methylpropyl)-1H-imidazole-2-thiol (7e). (76\%); orange crystals, mp $153^{\circ} \mathrm{C}$. El/MS, $m / z$ (RI\%): $342[\mathrm{M}]^{+}(51) .{ }^{1} \mathrm{H}$ NMR $\left(400 \mathrm{MHz}, \mathrm{CDCl}_{3}\right) \delta 0.97$ (d, J 6.6, 3H, $\left.\mathrm{CH}_{3}\right), 1.04$ (d, J 6.6, 3H, $\left.\mathrm{CH}_{3}\right)$, $1.93(\mathrm{ddt}, J 13.2,10.4,6.6,1 \mathrm{H}, \mathrm{CH}), 3.65\left(\mathrm{~m}, 4 \mathrm{H}, 2 \mathrm{CH}_{2}\right), 4.09(\mathrm{~s}, 2 \mathrm{H}, \mathrm{Fc}), 4.14(\mathrm{~s}, 2 \mathrm{H}, \mathrm{Fc}), 4.18(\mathrm{~s}, 5 \mathrm{H}, \mathrm{Fc}), 5.21(\mathrm{~d}$, J 10.3, 1H, CH), $5.72(\mathrm{~s}, 1 \mathrm{H}, \mathrm{NH}) .{ }^{13} \mathrm{C}$ NMR $\left(101 \mathrm{MHz}, \mathrm{CDCl}_{3}\right) \delta 20.3\left(\mathrm{CH}_{3}\right), 21.1\left(\mathrm{CH}_{3}\right), 27.6(\mathrm{CH}), 41.5\left(\mathrm{CH}_{2}\right), 43.4$ $\left(\mathrm{CH}_{2}\right), 52.7(\mathrm{CH}), 66.4\left(\mathrm{C}_{5} \mathrm{H}_{4}\right), 67.2\left(\mathrm{C}_{5} \mathrm{H}_{4}\right), 68.6\left(\mathrm{C}_{5} \mathrm{H}_{4}\right), 68.9\left(\mathrm{C}_{5} \mathrm{H}_{4}\right), 69.5\left(\mathrm{C}_{5} \mathrm{H}_{4}\right), 86.4\left(\right.$ ipso- $\left.\mathrm{C}_{5} \mathrm{H}_{4}\right), 182.5(\mathrm{C}=\mathrm{S})$. Calc. for $\mathrm{C}_{17} \mathrm{H}_{22} \mathrm{FeNS}_{2}$ : C, 59.65; H, 6.48; N, 8.18; Fe, 16.32; S, 9.37. Found: C, 59.58; H, 6.41; N, 8.50; Fe, 16.26.

4,5-Dihydro-1-(1-ferrocenyl(phenyl)methyl)-1H-imidazole-2-thiol (7f). (78\%); yellow crystals, mp $149^{\circ} \mathrm{C}$. $\mathrm{El} / \mathrm{MS}, \mathrm{m} / z$ (RI\%): $376[\mathrm{M}]^{+}(46) .{ }^{1} \mathrm{H}$ NMR $\left(400 \mathrm{MHz}, \mathrm{CDCl}_{3}\right) \delta 3.40\left(1 \mathrm{H}, \mathrm{m}, \mathrm{CH}_{2}\right), 3.54\left(2 \mathrm{H}, \mathrm{m}, \mathrm{CH}_{2}\right), 3.74(1 \mathrm{H}, \mathrm{m}$, $\left.\mathrm{CH}_{2}\right), 4.16(2 \mathrm{H}, \mathrm{s}, \mathrm{Fc}), 4.23(5 \mathrm{H}, \mathrm{s}, \mathrm{Fc}), 4.30(1 \mathrm{H}, \mathrm{s}, \mathrm{Fc}), 6.13(1 \mathrm{H}, \mathrm{s}, \mathrm{NH}), 7.02(1 \mathrm{H}, \mathrm{s}, \mathrm{CH}), 7.36(5 \mathrm{H}, \mathrm{m}, \mathrm{Ph}),{ }^{13} \mathrm{C}$ NMR $\left(101 \mathrm{MHz}, \mathrm{CDCl}_{3}\right) \delta 41.5\left(\mathrm{CH}_{2}\right), 45.1\left(\mathrm{CH}_{2}\right), 58.7(\mathrm{CH}), 67.7\left(\mathrm{C}_{5} \mathrm{H}_{4}\right), 68.1\left(\mathrm{C}_{5} \mathrm{H}_{4}\right), 68.2\left(\mathrm{C}_{5} \mathrm{H}_{4}\right), 69.2\left(\mathrm{C}_{5} \mathrm{H}_{4}\right)$, $69.3\left(\mathrm{C}_{5} \mathrm{H}_{5}\right), 86.2\left(\right.$ ipso- $\left.\mathrm{C}_{5} \mathrm{H}_{4}\right), 127.7\left(\mathrm{C}_{6} \mathrm{H}_{5}\right), 128.3\left(\mathrm{C}_{6} \mathrm{H}_{5}\right), 128.4\left(\mathrm{C}_{6} \mathrm{H}_{5}\right), 138.9$ (ipso- $\left.\mathrm{C}_{6} \mathrm{H}_{5}\right), 183.3$ (C=S). Calc. for $\mathrm{C}_{20} \mathrm{H}_{20} \mathrm{FeN}_{2} \mathrm{~S}: \mathrm{C}, 63.84 ; \mathrm{H}, 5.36 ; \mathrm{N}, 7.44 ; \mathrm{Fe}, 14.84 ; \mathrm{S}, 8.52$. Found: C, 63.88; H, 5.32; N, 7.42; Fe, 14.80.

\section{X-ray crystallography}

Single-crystal X-ray diffraction experiments for $\mathbf{5 f}, \mathbf{7 a}$ and $\mathbf{7 e}$ were carried out at $100 \mathrm{~K}$ on a Bruker APEX II diffractometer. Crystal data and experimental parameters are summarized in Table S1. The structures were solved by direct methods and refined by full-matrix last-squares technique for non-hydrogen atoms in the anisotropic approximation. All $\mathrm{H}$-atoms were placed in the geometrically calculated positions and included in the refinement using the riding model approximation with the $U_{\text {iso }}(H)=1.2 U_{\text {eq }}(C)$ for the methylene and $U_{\text {iso }}$ $(H)=1.5 U_{\text {eq }}(C)$ for methyl groups. All calculations calculations were carried out on IBM PC using SHELXTL program. ${ }^{25}$ Molecular graphics was produced by OLEX2 program. ${ }^{26}$ All measurements were carried out in of Center for molecular composition studies of INEOS RAS.

Crystal data for 5f, 7a and 7e were deposited in the Cambridge Crystallographic Data Centre (deposition numbers CCDC 1583214-1583216). These data are freely available from the Cambridge database upon request through the web page www.ccdc.cam.ac.uk/data_request/cif.

\section{Acknowledgements}

This work was financially supported by the Russian Science Foundation (Project No. 17-73-30036). E.Yu. Rogatkina is grateful for financial support Russian Foundation for Basic Research (Project No. 16-33-60163 mol_a_dk; synthesis of ferrocenylalkylated $N, S$-heterocycles).

\section{Supplementary Material}

${ }^{1} \mathrm{H},{ }^{13} \mathrm{C}$, and 2D NMR spectra of $5 \mathrm{c}$, feneral view of $\mathbf{5 f}, \mathbf{7 a}, \mathbf{e}$, the dimers in crystal packing of $\mathbf{7 a}$, the chains in crystal packing of 7e, main crystallographic data and refinement parameters for compounds $5 \mathbf{f}, \mathbf{7 a , e}$ (figures S1-S9 and table S1).

\section{References}

1. Neuse, E. W. J. Inorg. Organomet. Polymer. Mater. 2005, 15 (1), 3. https://doi.org/10.1007/s10904-004-2371-9 
2. Babin, V. N. ; Belousov, Yu A.; Borisov, V. I.; Gumenyuk, V. V. ; Nekrasov, Yu S.; Ostrovskaya, L. A.; Sviridova, I. K.; Sergeeva, N. S.; Simenel, A. A. and Snegur. L. V. Russ. Chem. Bull. 2014, 63, 2405. https://doi.org/10.1007/s11172-014-0756-7

3. Larik, F. A. ; Saeed, A. ; Fattah, T. A. ; Muqadar, U. ; Channar, P. A. Appl Organometal Chem. 2017, 31, 1. https://doi.org/10.1002/aoc.3664

4. Snegur, L.V.; Zykova, S.I.; Simenel, A.A.; Nekrasov, Y.S.; Starikova, Z.A.; Peregudova, S.M.; Ilyin, M.M.; Kachala, V.V.; Sviridova, I.K.; Sergeeva, N.S. Russ. Chem. Bull. 2013, 62, 2056.

https://doi.org/10.1007/s11172-013-0298-4

5. Snegur, L. V.; Nekrasov, Yu S.; Sergeeva, N. S.; Zhilina, Zh V.; Gumenyuk, V. V.; Starikova ,Z. A.; Simenel, A. A.; Morozova, N. B.; Sviridova, I. K.; and Babin, V. N. Appl. Organomet. Chem.2008, 22, 139. https://doi.org/10.1002/aoc.1362

6. Jaouen, G.; Vessières, A.; Top, S. Chem. Soc. Rev. 2015, 44, 8802. https://doi.org/10.1039/C5CS00486A

7. Rodionov, A.N.; Snegur, L.V.; Simenel, A.A.; Dobryakova, Y.V.; Markevich, V.A. Russ. Chem. Bull. 2017, 66, 136.

https://doi.org/10.1007/s11172-017-1711-1

8. Snegur, L.V.; Simenel, A.A.; Rodionov, A.N.; Boev, V.I. Russ. Chem. Bull. 2014, 63, 26. https://doi.org/10.1007/s11172-014-0390-4

9. Osipova, E.Yu.; Rodionov, A.N.; Belousov, Yu.A.; Il'in, M.M.; Nikolaev, A.L.; Gopin, A.V.; Mazina, S.E.; and Simenel, A.A. Russ.J. Org. Chem. 2016, 52 (1), 127.

https://doi.org/10.1134/S1070428016010243

10. Rodionov, A.N.; Gerasimova, M.D.; Osipova, E.Yu.; Korlyukov A.A.; Peregudov A.S.; Simenel A.A. Monatsh. Chem. 2017, 148, 925. https://doi.org/10.1007/s00706-016-1895-3

11. Osipova, E. Yu.; Ivanova, A. S.; Rodionov, A. N.; Korlyukov, A. A.; Arkhipov, D. E.; Simenel, A. A. Russ. Chem. Bull. 2016, 65, 2868. https://doi.org/10.1007/s11172-016-1670-y

12. Barnes, N.J. ; Barber, R.A. U.S. Patent 5726 129, 1998. https://patentimages.storage.googleapis.com/78/75/70/edf99be8f320a7/US5726129.pdf

13. Huang, Zh. ; Huang, M. ; Mi, Ch.; Wang, T. ; Chen, D. ; Teng, Y. Int. J. Mol. Sci. 2016, 17, 1330. https://doi.org/10.3390/ijms17081330

14. Sharma P. Ch., Kumar R., Chaudhary M., Sharma A., Rajak H. J. of Enzyme Inhib. Med. Chem. 2013, $28,1$. https://doi.org/10.3109/14756366.2011.611943

15. Kouge K., Koizumi T., Ishibashi N. and Okai H. Agric. Biol. Chem. 1987, 51 (7), 1941. http://doi.org/10.1271/bbb1961.51.1941

16. Kashyap, S.J.; Garg, V.K.; Sharma, P.K.; Kumar, N.; Dudhe, R.; Gupta J.K. Med. Chem. Res. 2012, 21, 2123. https://doi.org/10.1007/s00044-011-9685-2

17. Eicher, T.; Hauptmann, S. The Chemistry of Heterocycles, second ed., WileyVCH: Weinheim, 2003, p149. https://doi.org/10.1002/352760183X

18. Altintop, M.D.; Kaplancikli, Z.A.; Çiftçi, G.A.; Demirel R. Eur. J. Med. Chem. 2014, 74, 264; https://doi.org/10.1016/i.ejmech.2013.12.060

19. Fendt, M.; Endres, T. Neuroscience and Biobehavioral Rev. 2008, 32, 1259. https://doi.org/10.1016/j.neubiorev.2008.05.010 
20. Liu, J.C.; Liang, Y.; He, H.W. Chin. Chem. Lett. 2013, 24, 233. https://doi.org/10.1016/i.cclet.2013.01.036

21. Simenel, A.A.; Kuzmenko, Yu V.; Morozova, E.A.; Ilyin, M.M.; Gúnko, I.F.; Snegur, L.V. J. Organomet. Chem., $2003688,138$.

https://doi.org/10.1016/i.jorganchem.2003.08.039

22. Rodionov, A.N.; Zherebker, K.Ya.; Snegur, L.V.; Korlyukov, A.A.; Arhipov, D.E.; Peregudov, A.S.; Ilyin, M.M.; Ilyin Jr., M.M.; Nikitin, O.M.; Morozova, N.B.; Simenel, A.A. J. Organomet. Chem. 2015, 783, 83.

https://doi.org/10.1016/j.jorganchem.2015.01.031

23. Lindsay, J.K.; Hauser, C.R. J. Org. Chem. 1957, 22, 355. https://doi.org/10.1021/jo01355a001

24. Arimoto, F.S.; Haven ,A.S. J. Am. Chem. Soc. 1955, 77, 6295 https://doi.org/10.1021/ja01628a068

25. Sheldrick, G.M. Acta Crystallogr. 2008, A64, 112. https://doi.org/10.1107/S0108767307043930

26. Dolomanov, O.V.; Bourhis, L.J.; Gildea, R.J.; Howard, J.A.K.; Puschmann, H. J. Appl. Crystallogr. 2009, 42, 339; https://doi.org/10.1107/S0021889808042726 